\title{
Thermodynamics of chiral fermion system in a uniform magnetic field
}

\author{
Cheng Zhang, ${ }^{1, *}$ Ren-Hong Fang $\odot,{ }^{1, *}$ Jian-Hua Gao, ${ }^{2, *}$ and De-Fu Hou ${ }^{3, \dagger}$ \\ ${ }^{1}$ Key Laboratory of Particle Physics and Particle Irradiation (MOE), \\ Institute of Frontier and Interdisciplinary Science, Shandong University, \\ Qingdao, Shandong 266237, China \\ ${ }^{2}$ Shandong Provincial Key Laboratory of Optical Astronomy and Solar-Terrestrial Environment, \\ Institute of Space Sciences, Shandong University, Weihai, Shandong 264209, China \\ ${ }^{3}$ Institute of Particle Physics and Key Laboratory of Quark and Lepton Physics (MOS), \\ Central China Normal University, Wuhan 430079, China
}

(Received 10 June 2020; accepted 12 August 2020; published 8 September 2020)

\begin{abstract}
We construct the grand partition function of the system of chiral fermions in a uniform magnetic field from Landau levels, through which all thermodynamic quantities can be obtained. Making use of the AbelPlana formula, these thermodynamic quantities can be expanded as series with respect to a dimensionless variable $b=2 e B / T^{2}$. We find that the series expansions of the energy density, pressure, magnetization intensity, and magnetic susceptibility contain a singular term with $\ln b^{2}$, while the particle number density, entropy density, and heat capacity are power series of $b^{2}$. The asymptotic behaviors of these thermodynamic quantities in extreme conditions are also discussed.
\end{abstract}

DOI: 10.1103/PhysRevD.102.056004

\section{INTRODUCTION}

The properties of matter under an electromagnetic field have been studied extensively for years in many fields of physics. It is well known that a strong electric field can lead to pair production of fermions in QED vacuum, which is called the Schwinger mechanism [1-3]. Recently, the effect of a magnetic field on the Schwinger mechanism was studied through the approaches of an equal-time Wigner function and AdS/CFT correspondence [4-6]. In astronomy, compact stars, such as white dwarfs, neutron stars, and quark stars, often rotate very rapidly, which can produce a magnetic field as strong as $10^{12}-10^{15} \mathrm{G}[7,8]$. This strong magnetic field may have a great impact on the state of compact stars $[9,10]$. In high-energy physics, such as peripheral high-energy heavy ion collisions, a strong magnetic field in the collision region is also produced [11-14] and may induce the currents of charged particles along the direction of the magnetic field, which is called the chiral magnetic effect [15-19]. In condensed matter

\footnotetext{
*These authors contributed equally to this work.

Corresponding author. houdf@mail.ccnu.edu.cn

Corresponding author. gaojh@sdu.edu.cn

Published by the American Physical Society under the terms of the Creative Commons Attribution 4.0 International license. Further distribution of this work must maintain attribution to the author(s) and the published article's title, journal citation, and DOI. Funded by SCOAP ${ }^{3}$.
}

physics, the strong magnetic field can reduce a chiral condensate, which is called magnetic catalysis [20-25]. Meson condensation under the background of the magnetic field together with an electric field or rotation is also discussed in Refs. [26-29]. The magnetic field also has an important influence on the thermodynamics and transport properties of the system of massive fermions [30,31]. In relativistic hydrodynamics, the Wigner function approach is often used to study the hydrodynamics of a fermion system in a general electromagnetic field [17,32-35]. The chiral kinetic theory in the electromagnetic field is also studied recently $[36,37]$.

In this article, we study the influence of a magnetic field on the thermodynamics of the chiral fermion system, where we ignore the interaction among the fermions. Since the equations of motion for left-handed and right-handed fermions decouple, we will consider only the case of righthanded fermions in this article, and all results can be generalized to the left-handed case directly. In the previous work [38] by some of us, the electric current of the righthanded fermion system along the magnetic field, which is explained as the chiral magnetic effect, has been obtained through the ensemble average of normal ordering of the corresponding operator. In this article, we will use the method of the grand partition function instead, from which we can obtain all knowledge of the thermodynamic system. Through solving the stationary Schrödinger equation of a single right-handed fermion in a uniform magnetic field, we can obtain a series of Landau levels, from which we can construct the grand partition function. According to the standard procedure in quantum statistical mechanics, all 
thermodynamic quantities can be obtained by the grand partition function. In fact, for the thermodynamic system of massive fermions, the proper-time method is the most popular method to calculate the grand partition function [39-41]. However, for the thermodynamics of the chiral fermion system in this article, we will make use of the AbelPlana formula to calculate the grand partition function. In the expression of the grand partition function, there is a summation over all Landau levels, which is difficult to deal with analytically. Fortunately, there is an Abel-Plana formula which can transform the discrete summation into integrations. Then we can express the grand partition function as a two-dimensional integration, which can be expanded as a series with respect to a dimensionless variable $b=2 e B / T^{2}$, with $e$ the electric charge of the right-handed fermion, $B$ the magnetic field, and $T$ the temperature of the system. In the series, besides the terms with $b^{2 n}$, there is an additional singular term $b^{2} \ln b^{2}$, which indicates that the grand partition function is not analytic at $b=0$, leading to the nonanalyticity of some thermodynamic quantities at $b=0$. We investigate the asymptotic behaviors of these thermodynamic quantities in extreme conditions, such as weak and strong magnetic field limits and high- and lowtemperature limits. Our study of the effect of the magnetic field on the thermodynamics of the chiral fermion system may have an important theoretical meaning for research on the quark gluon plasma state which can be produced in highenergy heavy ion collisions.

The rest of this article is organized as follows. In Sec. II, the Landau levels of a single right-handed fermion in a uniform magnetic field are briefly listed. In Sec. III, we construct the grand partition function from Landau levels and express all thermodynamic quantities by the grand partition function. In Sec. IV, all thermodynamic quantities are expanded as series with respect to a dimensionless variable $b$. In Sec. V, we study the asymptotic behaviors of these thermodynamic quantities in extreme conditions. In Sec. VI, all elements of the energy-momentum tensor are calculated. This article is summarized in Sec. VII.

Throughout this article, we adopt natural units where $\hbar=c=k_{B}=1$. The convention for the metric tensor is $g^{\mu \nu}=\operatorname{diag}(+1,-1,-1,-1)$. We use the Heaviside-Lorentz convention for electromagnetism and the chiral representation for gamma matrices where $\gamma^{5}=\operatorname{diag}(-1,-1,+1,+1)$, which is the same as Peskin and Schroeder [42].

\section{LANDAU LEVELS FOR A SINGLE RIGHT-HANDED FERMION IN A UNIFORM MAGNETIC FIELD}

The Lagrangian of a chiral (massless) fermion field $\psi$ under the background of a uniform magnetic field $\boldsymbol{B}=\boldsymbol{B} \boldsymbol{e}_{z}$ is

$$
\mathcal{L}=\bar{\psi} i \gamma \cdot D \psi,
$$

where $D^{\mu}=\partial^{\mu}+i e A^{\mu}$, with $e$ the electric charge of the fermion and $A^{\mu}$ the gauge potential chosen as
$A^{\mu}=(0,0, B x, 0)$. In this article, we set $e B>0$ for simplicity. The results of all thermodynamic quantities in this article can be extended to the range $e B<0$.

In the chiral representation of gamma matrices, we can write $\psi=\left(\psi_{L}, \psi_{R}\right)^{T}$, where the two-component spinors $\psi_{L}$ and $\psi_{R}$ are called, respectively, left-handed and righthanded fermion fields. The Euler-Lagrange equation of the Lagrangian in Eq. (1) gives

$$
\begin{aligned}
i \frac{\partial}{\partial t} \psi_{L} & =-i \boldsymbol{\sigma} \cdot \boldsymbol{D} \psi_{L}, \\
i \frac{\partial}{\partial t} \psi_{R} & =i \boldsymbol{\sigma} \cdot \boldsymbol{D} \psi_{R},
\end{aligned}
$$

where $\boldsymbol{\sigma}=\left(\sigma^{1}, \sigma^{2}, \sigma^{3}\right)$ are Pauli matrices and $\boldsymbol{D}=$ $\left(-\partial_{x},-\partial_{y}+i e B x,-\partial_{z}\right)$. Since the equations of motion for $\psi_{L}$ and $\psi_{R}$ decouple, we discuss only the right-handed fermion field in this article. All results can be directly generalized to the left-handed case.

The stationary Schrödinger equation $i \boldsymbol{\sigma} \cdot \boldsymbol{D} \psi_{R}=E \psi_{R}$ gives a series of Landau levels and eigenfunctions as follows [38]:

$n=0, \quad E=k_{z}, \quad \psi_{R 0}\left(k_{y}, k_{z} ; \boldsymbol{x}\right)=\left(\begin{array}{c}\varphi_{0}(\xi) \\ 0\end{array}\right) \frac{1}{L} e^{i\left(y k_{y}+z k_{z}\right)}$, $n>0, \quad E=\lambda E_{n}\left(k_{z}\right)$,

$\psi_{R n \lambda}\left(k_{y}, k_{z} ; \boldsymbol{x}\right)=c_{n \lambda}\left(\begin{array}{c}\varphi_{n}(\xi) \\ i F_{n \lambda} \varphi_{n-1}(\xi)\end{array}\right) \frac{1}{L} e^{i\left(y k_{y}+z k_{z}\right)}$,

where $\lambda= \pm 1, \xi=\sqrt{e B} x-k_{y} / \sqrt{e B}, E_{n}\left(k_{z}\right)=\sqrt{2 n e B+k_{z}^{2}}$, $F_{n \lambda}=\left[k_{z}-\lambda E_{n}\left(k_{z}\right)\right] / \sqrt{2 n e B},\left|c_{n \lambda}\right|^{2}=1 /\left(1+F_{n \lambda}^{2}\right)$, and $\varphi_{n}(\xi)$ is the $n$th harmonic oscillator function along the $x$ axis whose center is $x=k_{y} / e B$. We have assumed that the eigenfunctions are set up in a box with sides of lengths $L$, i.e., $0<x, y, z<L$, and satisfy periodic boundary conditions in the $y$ axis and $z$ axis, i.e., $k_{y}=2 \pi n_{y} / L, k_{z}=$ $2 \pi n_{z} / L \quad\left(n_{y}, n_{z}=-\infty, \ldots, \infty\right)$. The condition that the center of the oscillation along the $x$ axis is inside the box leads to $0<n_{y}<e B L^{2} /(2 \pi)$. Since the energy level $E_{n}\left(k_{z}\right)$ is independent of $k_{y}$, the degeneracy of each Landau level is $e B L^{2} /(2 \pi)$.

\section{GRAND PARTITION FUNCTION AND THERMODYNAMIC QUANTITIES}

We consider a system of right-handed fermions in a uniform magnetic field $\boldsymbol{B}=B \boldsymbol{e}_{z}$, which is in equilibrium with a reservoir with temperature $T$ and chemical potential $\mu_{R}$. The interaction among the fermions in this system is ignored for simplicity. From the Landau levels for a single right-handed fermion in Sec. II, we can construct the grand partition function $\ln \Xi$ of this system as follows: 


$$
\begin{aligned}
\ln \Xi= & \sum_{k_{y}, k_{z}}\left[\theta\left(k_{z}\right) \ln \left(1+e^{a-\beta k_{z}}\right)+\theta\left(-k_{z}\right) \ln \left(1+e^{-a+\beta k_{z}}\right)\right] \\
& +\sum_{n=1}^{\infty} \sum_{k_{y}, k_{z}}\left[\ln \left(1+e^{a-\beta \sqrt{2 n e B+k_{z}^{2}}}\right)\right. \\
& \left.+\ln \left(1+e^{-a-\beta \sqrt{2 n e B+k_{z}^{2}}}\right)\right]
\end{aligned}
$$

where $\beta=1 / T$ and $a=\beta \mu_{R}$. The two theta functions $\theta\left(k_{z}\right)$ and $\theta\left(-k_{z}\right)$ in $\ln \Xi$ are necessary, as discussed in Ref. [38]. The vacuum terms in $\ln \Xi$ have been thrown away. The summations for $k_{y}$ and $k_{z}$ in Eq. (5) can be replaced by the degeneracy factor $e B L^{2} /(2 \pi)$ and the integral $(L / 2 \pi) \int d k_{z}$, respectively. Defining a dimensionless variable $b=2 e B \beta^{2}, \ln \Xi$ can be written as

$$
\ln \Xi=\frac{V}{\beta^{3}} g(a, b)
$$

where $V=L^{3}$ and $g(a, b)$ is defined as

$$
\begin{aligned}
g(a, b)= & \frac{b}{8 \pi^{2}} \int_{0}^{\infty} d s\left[\ln \left(1+e^{a-s}\right)+\ln \left(1+e^{-a-s}\right)\right] \\
& +\frac{b}{4 \pi^{2}} \int_{0}^{\infty} d s \sum_{n=1}^{\infty}\left[\ln \left(1+e^{a-\sqrt{n b+s^{2}}}\right)\right. \\
& \left.+\ln \left(1+e^{-a-\sqrt{n b+s^{2}}}\right)\right] .
\end{aligned}
$$

From the grand partition function $\ln \Xi$, the thermodynamic quantities of the system, such as particle number $N=V n$, energy $U=V \varepsilon$, pressure $p$, entropy $S=V s$, and magnetization intensity $M$, can be expressed as

$$
\begin{gathered}
N=\frac{\partial}{\partial a} \ln \Xi, \\
U=-\frac{\partial}{\partial \beta} \ln \Xi, \\
p=\frac{1}{\beta} \frac{\partial}{\partial V} \ln \Xi, \\
S=\ln \Xi+\beta U-a N, \\
M=\frac{1}{\beta} \frac{\partial}{\partial B}\left(\frac{\ln \Xi}{V}\right) .
\end{gathered}
$$

Making use of Eq. (6), all intensive quantities, such as particle number density $n$, energy density $\varepsilon$, pressure $p$, entropy density $s$, magnetization intensity $M$, magnetic susceptibility $\chi=\partial M / \partial B$, and heat capacity $c_{T}=\partial \varepsilon / \partial T$, can be expressed by $g(a, b)$ as follows:

$$
\begin{aligned}
& n=\frac{1}{\beta^{3}} \frac{\partial}{\partial a} g(a, b), \\
& \varepsilon=\frac{1}{\beta^{4}}\left(3-2 b \frac{\partial}{\partial b}\right) g(a, b) \\
& p=\frac{1}{\beta^{4}} g(a, b), \\
& s=\frac{1}{\beta^{3}}\left(4-a \frac{\partial}{\partial a}-2 b \frac{\partial}{\partial b}\right) g(a, b), \\
& M=\frac{2 e}{\beta^{2}} \frac{\partial}{\partial b} g(a, b), \\
& \chi=4 e^{2} \frac{\partial^{2}}{\partial b^{2}} g(a, b), \\
& c_{T}=\frac{1}{\beta^{3}}\left(12-3 a \frac{\partial}{\partial a}-10 b \frac{\partial}{\partial b}+2 a b \frac{\partial^{2}}{\partial a \partial b}+4 b^{2} \frac{\partial^{2}}{\partial b^{2}}\right) \\
& \times g(a, b) \text {. }
\end{aligned}
$$

\section{EXPANSIONS OF INTENSIVE QUANTITIES WITH RESPECT TO $b$}

To study the influence of the magnetic field on the thermodynamics of the right-handed fermion system, in this section we will expand all thermodynamic quantities as series with respect to $b=2 e B \beta^{2}$.

Defining an auxiliary function $f(a, x)$ as

$$
f(a, x)=\ln \left(1+e^{a-x}\right)+\ln \left(1+e^{-a-x}\right),
$$

then $g(a, b)$ in Eq. (7) becomes

$g(a, b)=\frac{b}{4 \pi^{2}} \int_{0}^{\infty} d s\left[\frac{1}{2} f(a, s)+\sum_{n=1}^{\infty} f\left(a, \sqrt{n b+s^{2}}\right)\right]$.

In Appendix A, we have proven that, when $-\pi<a<\pi$, the summation over Landau levels in the integrand in Eq. (21) can be transformed into integrations by the following Abel-Plana formula $[43,44]$ :

$$
\begin{aligned}
\frac{1}{2} \mathcal{F}(0)+\sum_{n=1}^{\infty} \mathcal{F}(n) \\
\quad=\int_{0}^{\infty} d t \mathcal{F}(t)+i \int_{0}^{\infty} d t \frac{\mathcal{F}(i t)-\mathcal{F}(-i t)}{e^{2 \pi t}-1} .
\end{aligned}
$$


Then $g(a, b)$ can be expressed as

$$
\begin{aligned}
g(a, b)= & \left(\frac{7 \pi^{2}}{360}+\frac{a^{2}}{12}+\frac{a^{4}}{24 \pi^{2}}\right) \\
& +\frac{b}{4 \pi^{2}} \times i \int_{0}^{\infty} d s \int_{0}^{\infty} d t \\
& \times \frac{f\left(a, \sqrt{i t b+s^{2}}\right)-f\left(a, \sqrt{-i t b+s^{2}}\right)}{e^{2 \pi t}-1},
\end{aligned}
$$

where the first term comes from the first integration on the right-hand side of Eq. (22):

$$
\int_{0}^{\infty} d s \int_{0}^{\infty} d t f\left(a, \sqrt{t+s^{2}}\right)=\frac{7 \pi^{4}}{90}+\frac{\pi^{2} a^{2}}{3}+\frac{a^{4}}{6} .
$$

In Appendix B, we obtained the series expansion of $g(a, b)$ at $b=0$ as follows:

$$
\begin{aligned}
g(a, b)= & \left(\frac{7 \pi^{2}}{360}+\frac{a^{2}}{12}+\frac{a^{4}}{24 \pi^{2}}\right)-\frac{b^{2} \ln b^{2}}{384 \pi^{2}} \\
& -\frac{b^{2}}{96 \pi^{2}} \ln \left(\frac{e^{1+C_{1}(a)}}{2 G^{6}}\right) \\
& -\frac{1}{2 \pi^{2}} \sum_{n=1}^{\infty} \frac{(4 n+1) ! !}{(4 n+4) ! !} \mathcal{B}_{2 n+2} C_{2 n+1}(a) b^{2 n+2},
\end{aligned}
$$

where $G=1.282427 \ldots$ is the Glaisher number, $\mathcal{B}_{n}$ are Bernoulli numbers, and $C_{2 n+1}(a)(n \geq 0)$ is

$$
\begin{aligned}
C_{2 n+1}(a)= & -\delta_{n, 0}+\frac{1}{(4 n+1) !} \int_{0}^{\infty} d y \ln y \frac{d^{4 n+1}}{d y^{4 n+1}} \\
& \times\left(\frac{1}{e^{y+a}+1}+\frac{1}{e^{y-a}+1}\right) .
\end{aligned}
$$

In the series of $g(a, b)$, besides the terms with $b^{2 n}$, there is also a single singular term $b^{2} \ln b^{2}$, which indicates that $g(a, b)$ is not analytic at $b=0$. When $a=0$, as calculated in Appendix D, the integration in Eq. (26) can be analytically integrated out:

$$
C_{2 n+1}(0)=(\ln 4+\gamma-1) \delta_{n, 0}+\frac{2 \zeta^{\prime}(-4 n)}{(4 n+1) !}\left(2^{4 n+1}-1\right) .
$$

Making use of Eqs. (13)-(19), all intensive quantities, such as particle number density $n$, energy density $\varepsilon$, pressure $p$, entropy density $s$, magnetization intensity $M$, magnetic susceptibility $\chi$, and heat capacity $c_{T}$, can be expressed as series of $b$ at $b=0$ in the following:

$$
\begin{aligned}
n \beta^{3}= & \left(\frac{a}{6}+\frac{a^{3}}{6 \pi^{2}}\right) \\
& -\frac{1}{2 \pi^{2}} \sum_{n=0}^{\infty} \frac{(4 n+1) ! !}{(4 n+4) ! !} \mathcal{B}_{2 n+2} C_{2 n+1}^{\prime}(a) b^{2 n+2},
\end{aligned}
$$

$$
\begin{aligned}
\varepsilon \beta^{4}= & \left(\frac{7 \pi^{2}}{120}+\frac{a^{2}}{4}+\frac{a^{4}}{8 \pi^{2}}\right)+\frac{b^{2} \ln b^{2}}{384 \pi^{2}}+\frac{b^{2}}{96 \pi^{2}} \ln \left(\frac{e^{2+C_{1}(a)}}{2 G^{6}}\right) \\
& +\frac{1}{2 \pi^{2}} \sum_{n=1}^{\infty} \frac{(4 n+1) ! !}{(4 n+4) ! !}(4 n+1) \mathcal{B}_{2 n+2} C_{2 n+1}(a) b^{2 n+2} \\
p \beta^{4}= & \left(\frac{7 \pi^{2}}{360}+\frac{a^{2}}{12}+\frac{a^{4}}{24 \pi^{2}}\right)-\frac{b^{2} \ln b^{2}}{384 \pi^{2}}-\frac{b^{2}}{96 \pi^{2}} \ln \left(\frac{e^{1+C_{1}(a)}}{2 G^{6}}\right) \\
& -\frac{1}{2 \pi^{2}} \sum_{n=1}^{\infty} \frac{(4 n+1) ! !}{(4 n+4) ! !} \mathcal{B}_{2 n+2} C_{2 n+1}(a) b^{2 n+2} \\
s \beta^{3}= & \left(\frac{7 \pi^{2}}{90}+\frac{a^{2}}{6}\right)+\frac{1+a C_{1}^{\prime}(a)}{96 \pi^{2}} b^{2} \\
& +\frac{1}{2 \pi^{2}} \sum_{n=1}^{\infty} \frac{(4 n+1) ! !}{(4 n+4) ! !} \mathcal{B}_{2 n+2}\left(4 n+a \frac{d}{d a}\right) \\
& \times C_{2 n+1}(a) b^{2 n+2}, \\
M \beta^{2} / e & =-\frac{b \ln b^{2}}{96 \pi^{2}}-\frac{b}{24 \pi^{2}} \ln \left(\frac{e^{5 / 4+C_{1}(a)}}{2 G^{6}}\right) \\
& -\frac{1}{2 \pi^{2}} \sum_{n=1}^{\infty} \frac{(4 n+1) ! !}{(4 n+2) ! !} \mathcal{B}_{2 n+2} C_{2 n+1}(a) b^{2 n+1}
\end{aligned}
$$

$$
\begin{aligned}
\chi / e^{2}= & -\frac{\ln b^{2}}{48 \pi^{2}}-\frac{1}{12 \pi^{2}} \ln \left(\frac{e^{7 / 4+C_{1}(a)}}{2 G^{6}}\right) \\
- & \frac{1}{2 \pi^{2}} \sum_{n=1}^{\infty} \frac{(4 n+1) ! !}{(4 n) ! !} \mathcal{B}_{2 n+2} C_{2 n+1}(a) b^{2 n}, \\
c_{T} \beta^{3}= & \left(\frac{7 \pi^{2}}{30}+\frac{a^{2}}{2}\right)-\frac{1+a C_{1}^{\prime}(a)}{96 \pi^{2}} b^{2} \\
& -\frac{1}{2 \pi^{2}} \sum_{n=1}^{\infty} \frac{(4 n+1) ! !}{(4 n+4) ! !}(4 n+1) \\
& \times \mathcal{B}_{2 n+2}\left(4 n+a \frac{d}{d a}\right) C_{2 n+1}(a) b^{2 n+2},
\end{aligned}
$$

where we have used the temperature factor $\beta$ and the electric charge $e$ to cancel the dimensions of all intensive quantities. We can see that in the expansions of energy density $\varepsilon$, pressure $p$, magnetization intensity $M$, and magnetic susceptibility $\chi$, besides the terms with $b^{n}$, there is also a term involving $\ln b^{2}$, which is singular at $b=0$. Nevertheless, the expansions of particle number density $n$, entropy density $s$, and heat capacity $c_{T}$ are just power series of $b^{2}$; i.e., they are analytic at $b=0$.

For the left-handed fermion system, we can simply replace $a=\beta \mu_{R}$ with $\beta \mu_{L}$ in the expressions obtained above for the right-handed fermion system, and all 
intensive quantities of the chiral fermion system can be obtained as a summation of the results of left-handed and right-handed fermion systems.

\section{EFFECT OF A MAGNETIC FIELD ON THERMODYNAMIC QUANTITIES}

In this section, we will investigate the effect of a magnetic field on the thermodynamics of the chiral fermion system. Since the dimensionless variables $a=\mu_{R} / T$ and $b=2 e B / T^{2}$ are proportional to the chemical potential $\mu_{R}$ and the magnetic field $B$, respectively, we will often use $a$ and $b$ instead of $\mu_{R}$ and $B$ to represent the chemical potential and the magnetic field in the following discussion. For a fixed chemical potential $a$, we take $Q(a, b)$ as the thermodynamic quantity with nonzero $b$ and $Q(a, 0)$ as the one with $b=0$.

According to Eqs. (13)-(19) and the integration expression of $g(a, b)$ in Eq. (23), we can plot the curves of all thermodynamic quantities as functions of $b$ and $a$. The asymptotic behaviors of all thermodynamic quantities as $b \rightarrow 0$ can be obtained directly by Eqs. (28)-(34). For the $b \rightarrow \infty$ limit, we must make use of the following asymptotic formula of $g(a, b)$, as calculated in Appendix E:

$$
\lim _{b \rightarrow \infty} g(a, b)=\frac{b}{48 \pi^{2}}\left(\pi^{2}+3 a^{2}\right) .
$$

Then Eqs. (13)-(19) can give the leading-order term for all thermodynamic quantities as $b \rightarrow \infty$.

\section{A. Particle number density}

The asymptotic behavior of particle number density ratio $n(a, b) / n(a, 0)$ as $b \rightarrow 0$ is

$$
\frac{n(a, b)}{n(a, 0)} \rightarrow 1-\frac{C_{1}^{\prime}(a) b^{2}}{16\left(\pi^{2} a+a^{3}\right)} .
$$

Making use of Eqs. (D9) and (C9) in the Appendixes, the coefficient of $b^{2}$ in Eq. (36) tends to be $-7 \zeta^{\prime}(-2) /\left(8 \pi^{2}\right)=$ 0.0027 as $a \rightarrow 0$ and tends to be zero as $a \rightarrow \infty$, which indicates that in a weak magnetic field limit the enhancement of the particle number density is smaller for a larger chemical potential. It is worth pointing out that Eq. (36) is consistent with the second-order result of particle number density in a weak electromagnetic field expansion by some of us in Ref. [35], where the Wigner function approach is used.

The asymptotic behavior of $n(a, b) / n(a, 0)$ as $b \rightarrow \infty$ is

$$
\frac{n(a, b)}{n(a, 0)} \rightarrow \frac{3 b}{4\left(\pi^{2}+a^{2}\right)}
$$

which increases linearly as $b \rightarrow \infty$ and increases more slowly for larger $a$.

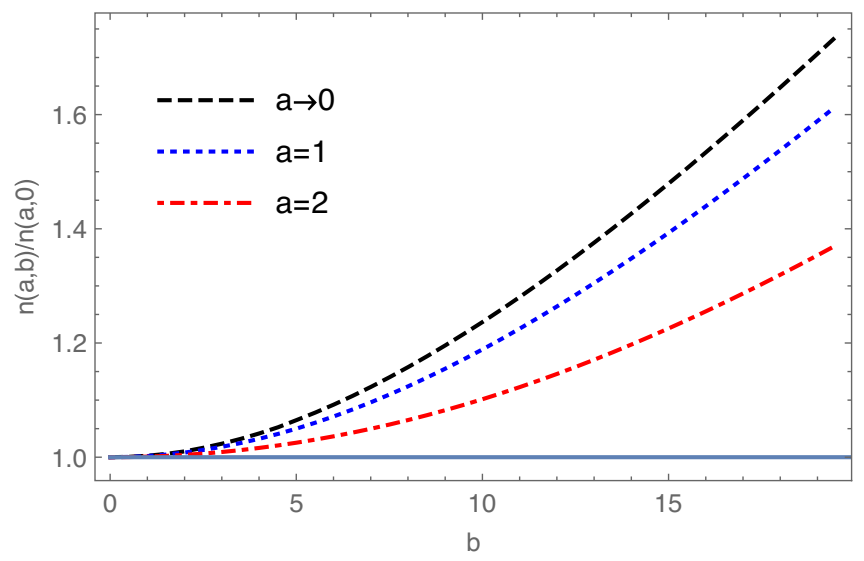

FIG. 1. Curves of particle number density ratio $n(a, b) / n(a, 0)$ with respect to $b$ for $a=1,2$ and $a \rightarrow 0$.

The asymptotic behavior of $n(a, b) / n(a, 0)$ as $a \rightarrow 0$ is listed as follows:

$$
\begin{aligned}
\lim _{a \rightarrow 0} \frac{n(a, b)}{n(a, 0)}= & 1+\frac{3 b}{\pi^{2}} \times i \int_{0}^{\infty} d s \int_{0}^{\infty} d t \frac{1}{e^{2 \pi t}-1} \\
& \times\left[\frac{e^{\sqrt{i t b+s^{2}}}}{\left(1+e^{\sqrt{i t b+s^{2}}}\right)^{2}}-\frac{e^{\sqrt{-i t b+s^{2}}}}{\left(1+e^{\sqrt{-i t b+s^{2}}}\right)^{2}}\right] .
\end{aligned}
$$

In Fig. 1, we plot the curves of $n(a, b) / n(a, 0)$ with respect to $b$ for $a=1,2$ and $a \rightarrow 0$. We can see that the existence of a magnetic field can considerably enhance the particle number density, and the enhancement is larger when $b$ is stronger and $a$ is smaller. The trends of the curves are consistent with our asymptotic analysis.

\section{B. Energy density and heat capacity}

From the expressions of the grand partition function $\ln \Xi$ and energy $U$ in Eqs. (5) and (9), we can express the energy density $\varepsilon$ by the following form:

$$
\begin{aligned}
\varepsilon= & \frac{1}{V} \sum_{k_{y}, k_{z}}\left(\frac{k_{z} \theta\left(k_{z}\right)}{e^{\beta\left(k_{z}-\mu_{R}\right)}+1}+\frac{\left(-k_{z}\right) \theta\left(-k_{z}\right)}{e^{\beta\left(-k_{z}+\mu_{R}\right)}+1}\right) \\
& +\frac{1}{V} \sum_{n=1}^{\infty} \sum_{k_{y}, k_{z}} E_{n}\left(k_{z}\right)\left[\frac{1}{e^{\beta\left[E_{n}\left(k_{z}\right)-\mu_{R}\right]}+1}+\frac{1}{e^{\beta\left[E_{n}\left(k_{z}\right)+\mu_{R}\right]}+1}\right] .
\end{aligned}
$$

The physical meaning of Eq. (39) is very clear: The whole energy of the system is jointly determined by the energy and the particle number of every quantum state and the degeneracy factor $e B L^{2} /(2 \pi)$ of every Landau level. As the magnetic field increases, the particle number of every state will decrease rapidly as $e^{-\sqrt{b}}$ due to Fermi-Dirac distribution; meanwhile, the product of the energy and the 


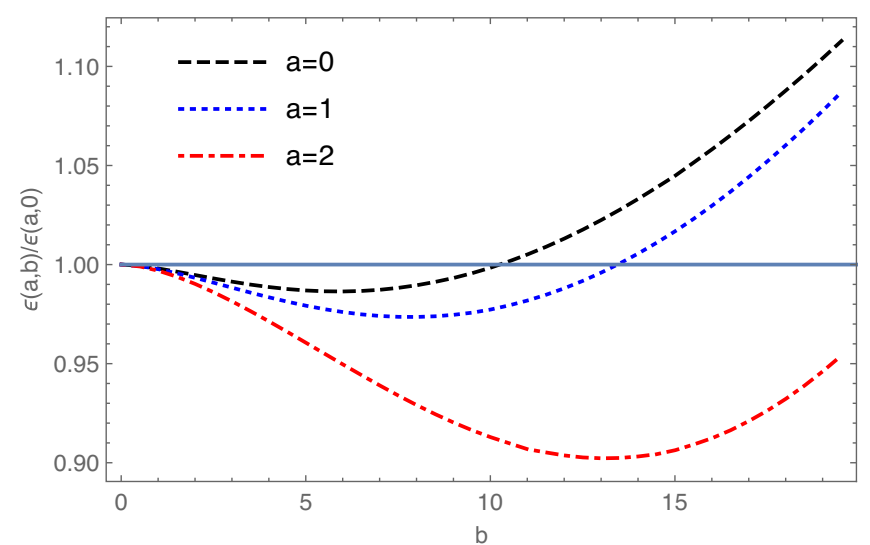

FIG. 2. Curves of energy density ratio $\varepsilon(a, b) / \varepsilon(a, 0)$ with respect to $b$ for $a=0,1,2$.

degeneracy of every Landau level increases as $b^{3 / 2}$, which indicates the existence of an extremum of energy density as $b$ increases.

In Fig. 2, we plot the curves of energy density ratio $\varepsilon(a, b) / \varepsilon(a, 0)$ with respect to $b$ for $a=0,1,2$. We can see that the curves decline first and then rise, leading to the appearance of an extremum as expected. When $a=0$, the extremum locates at about $(5.89,0.9864)$, and $\varepsilon(a, b) / \varepsilon(a, 0)=1$ at $b \approx 10.25$. When $a$ increases, the extremum moves to the lower right corner.

The asymptotic behavior of $\varepsilon(a, b) / \varepsilon(a, 0)$ as $b \rightarrow 0$ is

$$
\frac{\varepsilon(a, b)}{\varepsilon(a, 0)} \rightarrow 1+\frac{5 b^{2} \ln b^{2}}{16\left(7 \pi^{4}+30 \pi^{2} a^{2}+15 a^{4}\right)} .
$$

Since $b^{2} \ln b^{2}<0$ as $b<1$, the energy density in a weak magnetic field limit is smaller than the one without a magnetic field.

The asymptotic behavior of $\varepsilon(a, b)$ as $b \rightarrow \infty$ is

$$
\frac{\varepsilon(a, b)}{\varepsilon(a, 0)} \rightarrow \frac{5 \pi^{2}+15 a^{2}}{14 \pi^{4}+60 \pi^{2} a^{2}+30 a^{4}} \times b
$$

which increases linearly as $b \rightarrow \infty$ and increases more slowly for larger $a$. The trend of the curves in Fig. 2 is consistent with our asymptotic analysis.

If we take a high-temperature limit, i.e., $b \ll 1$ and $a \ll 1$, then the energy density becomes

$$
\varepsilon=\frac{7 \pi^{2}}{120} T^{4}+\frac{e^{2} B^{2}}{96 \pi^{2}} \ln \left(\frac{e^{2} B^{2}}{T^{4}}\right)
$$

We emphasize that the $\ln T$ term for the high-temperature limit in Eq. (42) is also consistent with the second-order result of energy density in Ref. [35].

If we take a low-temperature limit and $a=0$, then the energy density becomes

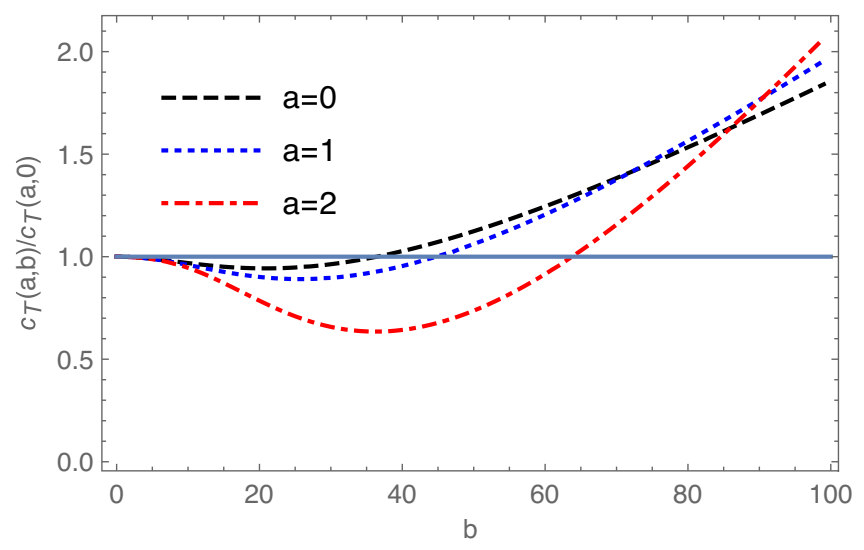

FIG. 3. Curves of heat capacity ratio $c_{T}(a, b) / c_{T}(a, 0)$ with respect to $b$ for $a=0,1,2$.

$$
\varepsilon= \begin{cases}\frac{1}{24} e B T^{2}, & B \neq 0, \\ \frac{7 \pi^{2}}{120} T^{4}, & B=0,\end{cases}
$$

which decreases to zero as $T \rightarrow 0$. We can see that the existence of a magnetic field makes the asymptotic behavior of the energy density become $T^{2}$ instead of $T^{4}$ as $T \rightarrow 0$.

In Fig. 3, we plot the curves of heat capacity ratio $c_{T}(a, b) / c_{T}(a, 0)$ with respect to $b$ for $a=0,1,2$, where there are also extrema. For the high-temperature limit, the heat capacity becomes

$$
c_{T}=\frac{7 \pi^{2}}{30} T^{3}-\frac{e^{2} B^{2}}{24 \pi^{2} T} .
$$

For a low-temperature limit with $a=0$, the heat capacity becomes

$$
c_{T}= \begin{cases}\frac{1}{12} e B T, & B \neq 0, \\ \frac{7 \pi^{2}}{30} T^{3}, & B=0,\end{cases}
$$

which tends to zero as $T \rightarrow 0$. Similar to the case of energy density, the existence of a magnetic field makes the asymptotic behavior of the heat capacity become $T$ instead of $T^{3}$ as $T \rightarrow 0$.

\section{Pressure and entropy density}

In Figs. 4 and 5, we plot the curves of pressure ratio $p(a, b) / p(a, 0)$ and entropy density ratio $s(a, b) / s(a, 0)$ with respect to $b$ for $a=0,1,2$, which are both increasing functions of $b$; i.e., the existence of the magnetic field can enhance the pressure and entropy density of the system.

For a high-temperature limit, the pressure and entropy density become, respectively,

$$
p=\frac{7 \pi^{2}}{360} T^{4}-\frac{e^{2} B^{2}}{96 \pi^{2}} \ln \left(\frac{e^{2} B^{2}}{T^{4}}\right)
$$




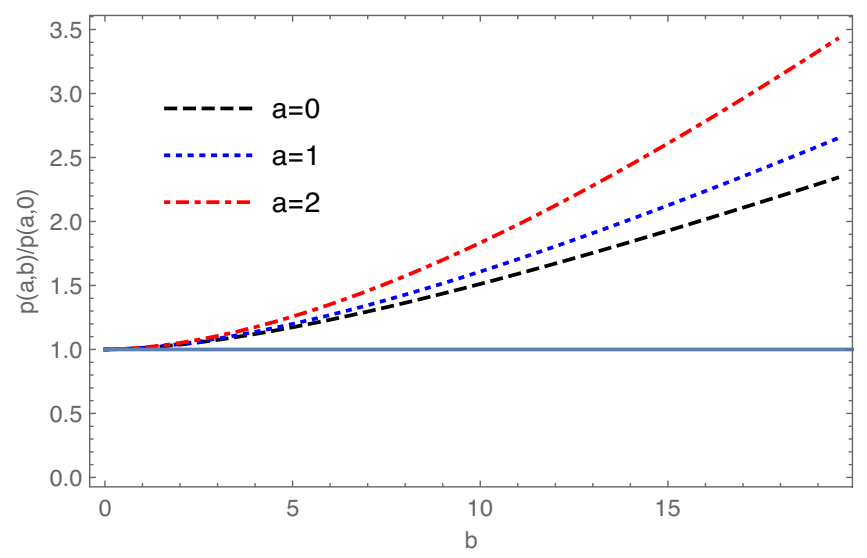

FIG. 4. Curves of pressure ratio $p(a, b) / p(a, 0)$ with respect to $b$ for $a=0,1,2$.

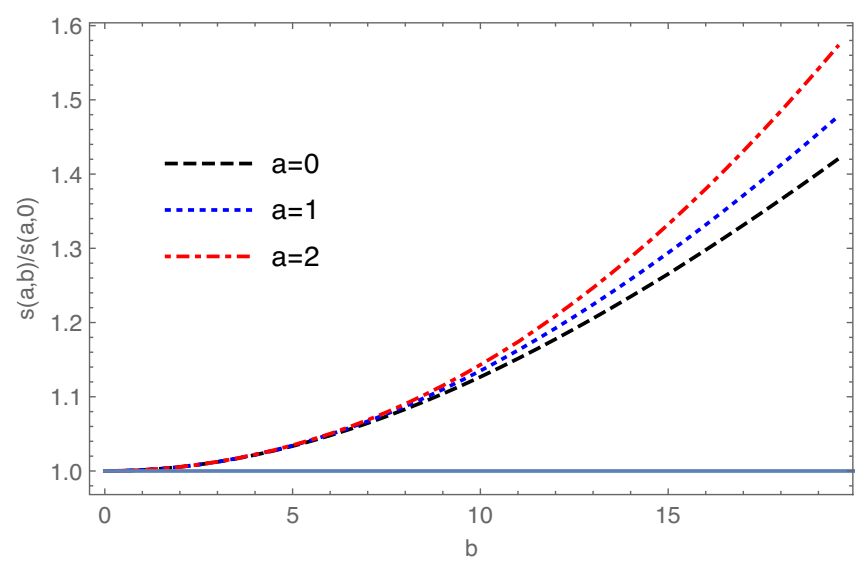

FIG. 5. Curves of entropy density ratio $s(a, b) / s(a, 0)$ with respect to $b$ for $a=0,1,2$.

and

$$
s=\frac{7 \pi^{2}}{90} T^{3}+\frac{e^{2} B^{2}}{24 \pi^{2} T}
$$

For a low-temperature limit with $a=0$, the pressure and entropy density become, respectively,

$$
p= \begin{cases}\frac{1}{24} e B T^{2}, & B \neq 0, \\ \frac{7 \pi^{2}}{360} T^{4}, & B=0\end{cases}
$$

and

$$
s= \begin{cases}\frac{1}{12} e B T, & B \neq 0, \\ \frac{7 \pi^{2}}{90} T^{3}, & B=0,\end{cases}
$$

which both tend to zero as $T \rightarrow 0$. Similar to the cases of energy density and heat capacity, the asymptotic behaviors

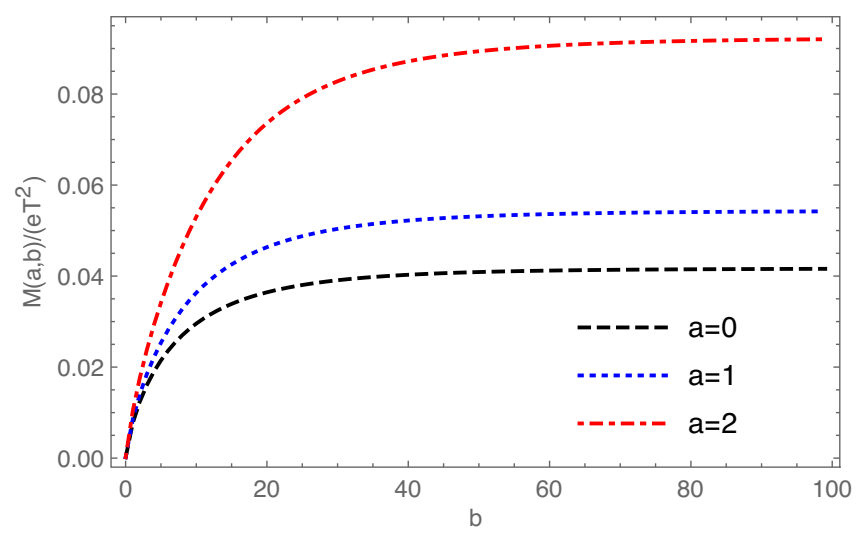

FIG. 6. Curves of magnetization intensity $M(a, b) /\left(e T^{2}\right)$ with respect to $b$ for $a=0,1,2$.

of the pressure and the entropy density as $T \rightarrow 0$ are also changed due to the existence of a magnetic field.

\section{Magnetization intensity and magnetic susceptibility}

In Fig. 6, we plot the curves of magnetization intensity $M(a, b)$ with respect to $b$ for $a=0,1,2$. As $b$ increases, the magnetization intensity increases rapidly from zero to saturation. The saturation value $M_{0}$ depends on the chemical potential as follows:

$$
M_{0}(a)=\frac{e T^{2}}{24}\left(1+\frac{3 a^{2}}{\pi^{2}}\right)
$$

Then we have $M_{0}(0)=0.042 e T^{2}, M_{0}(1)=0.054 e T^{2}$, and $M_{0}(2)=0.092 e T^{2}$, which are consistent with the saturation values in Fig. 6.

Since the magnetization intensity $M(a, b)$ becomes saturated as $b \rightarrow \infty$, the magnetic susceptibility $\chi(a, b)$ tends to zero as $b \rightarrow \infty$. In Fig. 7, we plot the curves of magnetic susceptibility $\chi(a, b)$ with respect to $b$ for $a=0$, 1,2 , where the curves tend to zero as $b \rightarrow \infty$ and tend to

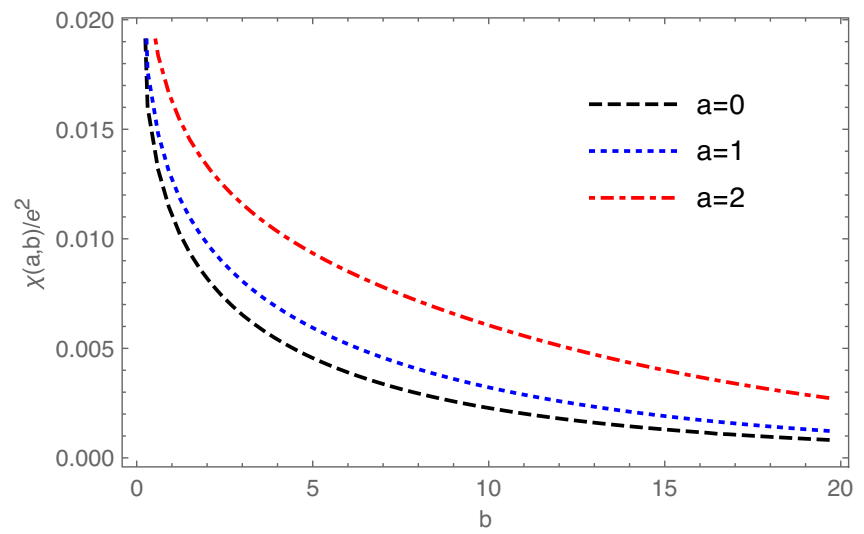

FIG. 7. Curves of magnetic susceptibility $\chi(a, b) / e^{2}$ with respect to $b$ for $a=0,1,2$. 
divergent as $b \rightarrow 0$. In fact, the magnetic susceptibility in a weak magnetic field limit or high-temperature limit becomes

$$
\chi=-\frac{e^{2}}{48 \pi^{2}} \ln \left(\frac{e^{2} B^{2}}{T^{4}}\right),
$$

which is logarithmically divergent as $B \rightarrow 0$ or $T \rightarrow \infty$. The $\ln T$ term in Eq. (51) was also derived in a recent article [45], where the authors have calculated the hightemperature expansion of the magnetic susceptibility of QCD matter with a physical quark mass, and the leadingorder term is just $\ln T$ with the same coefficient as ours.

In a low-temperature limit, i.e., $b \rightarrow \infty$, the magnetic susceptibility tends to zero, indicating that the system reaches the magnetic saturation state. This is consistent with the numerical result of the lattice method for a QCD matter system $[45,46]$.

In Fig. 7, we can see that $\chi$ is always positive, so the chiral fermion system is a paramagnetic system.

\section{E. A brief summary}

We have studied the effect of a magnetic field on the thermodynamics of the right-handed fermion system. All results calculated above can be directly generalized to the left-handed case. In Table I, we list the leading-order terms of all intensive quantities of the chiral fermion system as $b \rightarrow 0$ and $b \rightarrow \infty$, with $a=0$.

In summary, we conclude that the magnetic field has great influence on the thermodynamics of the chiral fermion system. In high-energy heavy ion collisions, a new matter state "quark gluon plasma" can be created [47-49], where the quarks and gluons are deconfined from the interior of the hadrons due to the high temperature and the chiral symmetry may be recovered [50,51]. For peripheral collisions, a huge magnetic field will be produced in the collision region [11-14], which may considerably change the properties of the deconfined matter state and, hence, influence the distributions in phase space of the final particles from the collision.

\section{ENERGY-MOMENTUM TENSOR}

In this section, we will show how the nonzero components of $T^{\mu \nu}$ of the right-handed fermion system are related to $g(a, b)$. The 00 component of $T^{\mu \nu}$ is just the energy density $\varepsilon$, which has been calculated through the grand partition function in Secs. III and IV. Now we want to calculate other components of $T^{\mu \nu}$.

Since the thermodynamic system is located in a uniform magnetic field $\boldsymbol{B}=B \boldsymbol{e}_{z}$, all thermodynamic quantities will be unchanged under the rotation along the $z$ axis. The rotation by an angle $\phi$ along the $z$ axis can be represented by the following matrix:

$$
\Lambda_{\nu}^{\mu}(\phi)=\left(\begin{array}{cccc}
1 & 0 & 0 & 0 \\
0 & \cos \phi & -\sin \phi & 0 \\
0 & \sin \phi & \cos \phi & 0 \\
0 & 0 & 0 & 1
\end{array}\right)
$$

In order to keep $T^{\mu \nu}$ unchanged under the rotation matrix $\Lambda_{\nu}^{\mu}(\phi)$ for any angle $\phi$, i.e., $T^{\mu \nu}=\Lambda_{\alpha}^{\mu}{ }_{\alpha}{ }^{\nu}{ }_{\beta} T^{\alpha \beta}$, the components of $T^{\mu \nu}$ must satisfy these conditions:

$$
T^{01}=T^{02}=T^{12}=T^{13}=T^{23}=0, \quad T^{11}=T^{22} ;
$$

i.e., the nonzero components are $T^{00}, T^{33}, T^{11}=T^{22}$, and $T^{03}$. According to Eq. (3), the trace of $T^{\mu \nu}$ is zero, so the independent components are $T^{00}, T^{11}$, and $T^{03}$.

We cannot directly calculate $T^{11}$ and $T^{03}$ by the grand partition function. Alternatively, we appeal to the ensemble average approach, in which all macroscopic quantities are the ensemble average of normal ordering of the corresponding operators. The symmetric and gauge-invariant energy-momentum tensor is

$$
T^{\mu \nu}=\frac{1}{2}\left\langle: \psi_{R}^{\dagger} i \sigma^{\mu} D^{\nu} \psi_{R}+\psi_{R}^{\dagger} i \sigma^{\nu} D^{\mu} \psi_{R}:\right\rangle,
$$

where $\sigma^{\mu}=(1, \boldsymbol{\sigma}), D^{\mu}=\left(\partial_{t},-\partial_{x},-\partial_{y}+i e B x,-\partial_{z}\right)$, the angular brackets mean ensemble average, and the double dots enclosing the field operators mean normal ordering as adopted in Refs. [38,52]. In Appendix F, we have compared the results of normal ordering and un-normal ordering descriptions. For the un-normal ordering description, one should add the vacuum term to the grand partition function in Eq. (5). The un-normal ordering description is adopted in Ref. [35], where the contribution of a vacuum term is considered, resulting in a regular form of $e B$, i.e., $(e B)^{2} \ln \left(\Lambda^{2} / T^{2}\right)$, with $\Lambda$ the renormalization scale, and the logarithmic term of $e B$ disappears. For the QED case, the vacuum term contributes a similar logarithmic term

\begin{tabular}{|c|c|c|c|c|c|c|c|c|}
\hline Quantities & $n$ & $\varepsilon \times \beta^{4}$ & $p \times \beta^{4}$ & $s \times \beta^{3}$ & $M \times \beta^{2}$ & $\chi$ & $c_{T} \times \beta^{3}$ & $T^{11} \times \beta^{4}$ \\
\hline$b \rightarrow 0$ & 0 & $\frac{7 \pi^{2}}{60}+\frac{b^{2} \ln b^{2}}{192 \pi^{2}}$ & $\frac{7 \pi^{2}}{180}-\frac{b^{2} \ln b^{2}}{192 \pi^{2}}$ & $\frac{7 \pi^{2}}{45}+\frac{b^{2}}{48 \pi^{2}}$ & $-\frac{e b \ln b^{2}}{48 \pi^{2}}$ & $-\frac{e^{2} \ln b^{2}}{24 \pi^{2}}$ & $\frac{7 \pi^{2}}{15}-\frac{b^{2}}{48 \pi^{2}}$ & $\frac{7 \pi^{2}}{180}+\frac{b^{2} \ln b^{2}}{192 \pi^{2}}$ \\
\hline$b \rightarrow \infty$ & 0 & $\frac{b}{24}$ & $\frac{b}{24}$ & $\frac{b}{12}$ & $\frac{e}{12}$ & 0 & $\frac{b}{12}$ & 0 \\
\hline
\end{tabular}
of the renormalization scale $\Lambda$, which is related to the beta function of the theory [53].

TABLE I. Leading-order terms of all intensive quantities of the chiral fermion system as $b \rightarrow 0$ and $b \rightarrow \infty$ (with $a=0$ ). 
In the following, we will calculate $T^{00}, T^{11}$, and $T^{03}$ through ensemble average with the normal ordering description. In order to calculate Eq. (54), the field operator $\psi_{R}(\boldsymbol{x})$ must be expanded by the orthonormal and complete eigenfunctions in Eq. (4) as follows [38]:

$$
\begin{aligned}
\psi_{R}(\boldsymbol{x})= & \sum_{k_{y}, k_{z}}\left[a_{0}\left(k_{y}, k_{z}\right) \theta\left(k_{z}\right) \psi_{R 0}\left(k_{y}, k_{z} ; \boldsymbol{x}\right)\right. \\
& \left.+b_{0}^{\dagger}\left(k_{y}, k_{z}\right) \theta\left(-k_{z}\right) \psi_{R 0}\left(k_{y}, k_{z} ; \boldsymbol{x}\right)\right] \\
& +\sum_{n, k_{y}, k_{z}}\left[a_{n}\left(k_{y}, k_{z}\right) \psi_{R n+}\left(k_{y}, k_{z} ; \boldsymbol{x}\right)\right. \\
& \left.+b_{n}^{\dagger}\left(k_{y}, k_{z}\right) \psi_{R n-}\left(k_{y}, k_{z} ; \boldsymbol{x}\right)\right],
\end{aligned}
$$

where $a_{n}, a_{n}^{\dagger}, b_{n}$, and $b_{n}^{\dagger}$ are annihilation and creation operators for fermions and antifermions. As calculated in Ref. [38], the ensemble average of normal ordering for $a_{n}^{\dagger} a_{n}$ and $b_{n} b_{n}^{\dagger}$ is

$$
\begin{array}{r}
\left\langle: \theta\left(k_{z}\right) a_{0}^{\dagger}\left(k_{y}, k_{z}\right) a_{0}\left(k_{y}, k_{z}\right):\right\rangle=\frac{\theta\left(k_{z}\right)}{e^{\beta\left(k_{z}-\mu_{R}\right)}+1}, \\
\left\langle: \theta\left(-k_{z}\right) b_{0}\left(k_{y}, k_{z}\right) b_{0}^{\dagger}\left(k_{y}, k_{z}\right):\right\rangle=-\frac{\theta\left(-k_{z}\right)}{e^{\beta\left(-k_{z}+\mu_{R}\right)}+1}, \\
\left\langle: a_{n}^{\dagger}\left(k_{y}, k_{z}\right) a_{n}\left(k_{y}, k_{z}\right):\right\rangle=\frac{1}{e^{\beta\left[E_{n}\left(k_{z}\right)-\mu_{R}\right]}+1}, \\
\left\langle: b_{n}\left(k_{y}, k_{z}\right) b_{n}^{\dagger}\left(k_{y}, k_{z}\right):\right\rangle=-\frac{1}{e^{\beta\left[E_{n}\left(k_{z}\right)+\mu_{R}\right]}+1} .
\end{array}
$$

Substituting Eq. (55) into Eq. (54) and making use of Eqs. (56)-(59) gives

$T^{03}=\frac{e B}{(2 \pi)^{2}} \int_{0}^{\infty} d k_{z} k_{z}\left(\frac{1}{e^{\beta\left(k_{z}-\mu_{R}\right)}+1}+\frac{1}{e^{\beta\left(k_{z}-\mu_{R}\right)}+1}\right)$,

$$
\begin{aligned}
T^{11}= & \frac{(e B)^{2}}{2 \pi^{2}} \int_{0}^{\infty} d k_{z} \\
& \times \sum_{n=1}^{\infty} \frac{n}{E_{n}}\left(\frac{1}{e^{\beta\left[E_{n}\left(k_{z}\right)-\mu\right]}+1}+\frac{1}{e^{\beta\left[E_{n}\left(k_{z}\right)+\mu\right]}+1}\right) .
\end{aligned}
$$

We can see that only the lowest Landau level $(n=0)$ contributes to $T^{03}$, while $T^{11}$ comes from higher Landau levels $(n>0)$. Further calculation gives

$$
\begin{gathered}
T^{03}=\frac{b}{8 \pi^{2} \beta^{4}}\left(\frac{\pi^{2}}{6}+\frac{a^{2}}{2}\right), \\
T^{11}=\frac{1}{\beta^{4}}\left(1-b \frac{\partial}{\partial b}\right) g(a, b) .
\end{gathered}
$$

We can see that $T^{03}$ is linear only in $b$. In fact, the space component of the particle number current is $a b /\left(8 \pi^{2} \beta^{3}\right)$, also linear in $b$ as calculated in Ref. [38], which is called the chiral magnetic effect. When $a=0$ (i.e., the chemical potential is zero), the particle number current disappears, but a nonzero term $e B T^{2} / 24$ remains in $T^{03}$, which is reasonable since the same number of fermions and antifermions move in the same direction along the $z$ axis as discussed in Ref. [38], resulting in a zero value of the net particle number current and a nonzero value of the energy current.

For the left-handed fermion system, $T^{11}$ can be obtained by simply replacing $a=\beta \mu_{R}$ with $\beta \mu_{L}$ in Eq. (63). However, $T^{03}$ for the left-handed fermion system can be obtained by space inversion in Eq. (62), and the result is

$$
-\frac{b}{8 \pi^{2} \beta^{4}}\left(\frac{\pi^{2}}{6}+\frac{\beta^{2} \mu_{L}^{2}}{2}\right) \text {. }
$$

The total $T^{03}$ of the chiral fermion system is the summation of the results of left-handed and right-handed fermion systems, and the result is $e B \mu \mu_{5} /\left(2 \pi^{2}\right)$ with $\mu=$ $\frac{1}{2}\left(\mu_{R}+\mu_{L}\right)$ and $\mu_{5}=\frac{1}{2}\left(\mu_{R}-\mu_{L}\right)$, which is consistent with Ref. [17].

From the series expansion of $g(a, b)$, we can obtain

$$
\begin{aligned}
T^{11}= & \frac{1}{4 \pi^{2} \beta^{4}}\left[\left(\frac{7 \pi^{4}}{90}+\frac{\pi^{2} a^{2}}{3}+\frac{a^{4}}{6}\right)\right. \\
& +\frac{b^{2} \ln b^{2}}{96}+\frac{b^{2}}{24} \ln \left(\frac{e^{3 / 2+C_{1}(a)}}{2 G^{6}}\right) \\
& \left.+\sum_{n=1}^{\infty} \frac{(4 n+1) ! !}{(4 n+4) ! !}(4 n+2) \mathcal{B}_{2 n+2} C_{2 n+1}(a) b^{2 n+2}\right] .
\end{aligned}
$$

Since $g_{\mu \nu} T^{\mu \nu}=0$ and $T^{11}=T^{22}$, the result for $T^{33}$ is

$$
T^{33}=T^{00}-2 T^{11}=\frac{g(a, b)}{\beta^{4}}=p,
$$

so we conclude that $T^{33}$ is just the pressure $p$ of the system.

\section{SUMMARY}

In this article, we have studied the thermodynamics of the chiral fermion system in a uniform magnetic field, where we ignored the interaction among all fermions. Since the equations of motion for left-handed fermions and righthanded fermions decouple, we did all the calculations for the case of the right-handed fermion, which can be generalized to the left-handed case directly. From the Landau levels of a single right-handed fermion in a uniform magnetic field, we construct the grand partition function of this thermodynamic system, through which all intensive 
quantities can be obtained as a summation over all Landau levels. Making use of the Abel-Plana formula, the summation over all Landau levels can be transformed into integrations, which can be more readily dealt with analytically. We expanded these thermodynamic quantities as series with respect to a dimensionless variable $b=2 e B / T^{2}$. We find that the series expansions of energy density, pressure, magnetization intensity, and magnetic susceptibility contain a singular term with $\ln b^{2}$, which indicates that these thermodynamic quantities are not analytic at $b=0$. Meanwhile, the series expansions of particle number density, entropy density, and heat capacity are power series of $b^{2}$, which indicates the analyticity of them at $b=0$. We plot the curves of these thermodynamic quantities with respect to $b$ with zero and finite chemical potentials, respectively, and discuss the asymptotic behaviors of these quantities in strong and weak magnetic field limits and low- and high-temperature limits. All elements of the energy-momentum tensor are also calculated. We conclude that the magnetic field can have an important influence on the thermodynamics of the chiral fermion system, which may be helpful to study the properties of the quark gluon plasma state created in high-energy heavy ion collisions.

\section{ACKNOWLEDGMENTS}

We are grateful to Michael Peskin and Hai-Cang Ren for helpful discussion. This work was supported by the National Natural Science Foundation of China under Grants No. 11890713, No. 11735007, and No. 11890711.

\section{APPENDIX A: ABEL-PLANA FORMULA}

In mathematics, the Abel-Plana formula is a summation formula which was discovered by Abel (1823) and Plana (1820) independently [44]. It states that, if a function $\mathcal{F}(z)$ is analytic at $\operatorname{Re} z \geq 0$ and $\mathcal{F}(z) \rightarrow 0$ as $|z| \rightarrow \infty$ along the positive real axis, then

$\frac{1}{2} \mathcal{F}(0)+\sum_{n=1}^{\infty} \mathcal{F}(n)=\int_{0}^{\infty} d t \mathcal{F}(t)+i \int_{0}^{\infty} d t \frac{\mathcal{F}(i t)-\mathcal{F}(-i t)}{e^{2 \pi t}-1}$.

In Sec. IV, we met with the following function:

$g(a, b)=\frac{b}{4 \pi^{2}} \int_{0}^{\infty} d s\left[\frac{1}{2} f(a, s)+\sum_{n=1}^{\infty} f\left(a, \sqrt{n b+s^{2}}\right)\right]$,

where $b>0$ and $f(a, x)$ is defined as

$$
f(a, x)=\ln \left(1+e^{a-x}\right)+\ln \left(1+e^{-a-x}\right) .
$$

We can see that the function $\mathcal{F}(z)=f\left(a, \sqrt{z b+s^{2}}\right)$ satisfies $\mathcal{F}(z) \rightarrow 0$ as $|z| \rightarrow \infty$ along the positive real axis. In order to apply the Abel-Plana formula to the summation in the integrand in Eq. (A2), the function $\mathcal{F}(z)=$ $f\left(a, \sqrt{z b+s^{2}}\right)$ must be analytic at $\operatorname{Re} z \geq 0$. The possible poles appear in $\mathcal{F}(z)$ when

$$
1+e^{ \pm a-\sqrt{z b+s^{2}}}=0
$$

which implies

$$
\begin{aligned}
z= & \frac{1}{b}\left[a^{2}-(2 n+1)^{2} \pi^{2}-s^{2} \mp i(4 n+2) \pi a\right] \\
& (n=0, \pm 1, \pm 2, \ldots) .
\end{aligned}
$$

In order to make $\mathcal{F}(z)$ analytic at $\operatorname{Re} z \geq 0$, the poles in Eq. (A5) must lie in the range $\operatorname{Re} z<0$; i.e., the inequation $a^{2}-(2 n+1)^{2} \pi^{2}-s^{2}<0$ must be satisfied for any $n$ and $s$, which indicates $-\pi<a<\pi$.

\section{APPENDIX B: EXPANSION OF $g(a, b)$ AT $b=0$}

Define an auxiliary function $F(a, x)$ as

$$
F(a, x)=\int_{0}^{\infty} d s f\left(a, \sqrt{x^{2}+s^{2}}\right)
$$

where $f(a, x)=\ln \left(1+e^{a-x}\right)+\ln \left(1+e^{-a-x}\right)$ as defined in Eq. (20). Then $g(a, b)$ in Eq. (23) becomes

$$
\begin{aligned}
g(a, b)= & \left(\frac{7 \pi^{2}}{360}+\frac{a^{2}}{12}+\frac{a^{4}}{24 \pi^{2}}\right) \\
& +\frac{b}{4 \pi^{2}} \times i \int_{0}^{\infty} d t \frac{F(a, \sqrt{i t b})-F(a, \sqrt{-i t b})}{e^{2 \pi t}-1} .
\end{aligned}
$$

Now we will expand $F(a, x)$ at $x=0$ in the following. By the variable transformation $y=\sqrt{x^{2}+s^{2}}, F(a, x)$ can be written as

$$
F(a, x)=\int_{|x|}^{\infty} d y \frac{y}{\sqrt{y^{2}-x^{2}}} f(a, y) .
$$

The factor $y / \sqrt{y^{2}-x^{2}}$ in the integrand in $F(a, x)$ can be replaced by the following Taylor expansion:

$$
\frac{y}{\sqrt{y^{2}-x^{2}}}=\sum_{n=0}^{\infty} \frac{(2 n-1) ! !}{(2 n) ! !} \frac{x^{2 n}}{y^{2 n}},
$$

where we have defined $(-1) ! !=0 ! !=1$. Then $F(a, x)$ becomes 


$$
F(a, x) \equiv \sum_{n=0}^{\infty} \frac{(2 n-1) ! !}{(2 n) ! !} x^{2 n} d_{n}(a, x)
$$

where we have defined $d_{n}(a, x)$ as

$$
d_{n}(a, x)=\int_{|x|}^{\infty} d y \frac{1}{y^{2 n}} f(a, y)
$$

The derivative of $d_{n}(a, x)$ with respect to $x$ is

$$
d_{n}^{\prime}(a, x)=-\frac{|x|}{x^{2 n+1}}[f(a, x)+x]+\frac{1}{x^{2 n-1}} .
$$

Note that the factor $f(a, x)+x$ in Eq. (B7) is an even function of $x$ and can be expanded at $x=0$ as follows:

$$
f(a, x)+x=\sum_{k=0}^{\infty} c_{k}(a) x^{2 k}
$$

From $d_{n}^{\prime}(a, x)$, we can derive $d_{n}(a, x)$ as

$$
\begin{aligned}
d_{n}(a, x)= & -|x| \sum_{k=0}^{\infty} \frac{c_{k}(a)}{2 k-2 n+1} x^{2 k-2 n} \\
& + \begin{cases}C_{1}(a)+\frac{1}{2} \ln x^{2}, & n=1, \\
C_{n}(a)-\frac{1}{2(n-1)} x^{2-2 n}, & n \neq 1,\end{cases}
\end{aligned}
$$

where $C_{n}(a)$ are independent of $x$, which will be calculated later.

Making use of the following two identities:

$$
\begin{gathered}
\sum_{n=2}^{\infty} \frac{(2 n-1) ! !}{(2 n) ! !} \frac{1}{2(n-1)}=\frac{1}{2} \ln 2+\frac{1}{4}, \\
\sum_{n=0}^{\infty} \frac{(2 n-1) ! !}{(2 n) ! !} \frac{1}{2 n-2 k-1}=0 \quad(k=0,1,2, \ldots),
\end{gathered}
$$

we can obtain $F(a, x)$ in Eq. (B5) as

$$
\begin{aligned}
F(a, x)= & C_{0}(a)+\frac{1}{4} x^{2} \ln x^{2}+\left(\frac{1}{4}-\frac{1}{2} \ln 2+\frac{1}{2} C_{1}(a)\right) x^{2} \\
& +\sum_{n=2}^{\infty} \frac{(2 n-1) ! !}{(2 n) ! !} C_{n}(a) x^{2 n} .
\end{aligned}
$$

In the following, we will extract $C_{n}(a)$ in $d_{n}(a, x)$. When $n=0$, we have $C_{0}(a)=d_{0}(a, 0)=\pi^{2} / 6+a^{2} / 2$. When $n>0$, through integration by parts we have

$$
\begin{aligned}
d_{n}(a, x)= & \sum_{k=0}^{2 n-2} \frac{(2 n-k-2) !}{(2 n-1) !} \frac{1}{x^{2 n-k-1}} \frac{d^{k}}{d x^{k}} f(a, x) \\
& -\frac{\ln x}{(2 n-1) !} \frac{d^{2 n-1}}{d x^{2 n-1}} f(a, x) \\
& -\frac{1}{(2 n-1) !} \int_{x}^{\infty} d y \ln y \frac{d^{2 n}}{d y^{2 n}} f(a, y) .
\end{aligned}
$$

Making use of Eq. (B8), we obtain

$$
C_{n}(a)=-\delta_{n, 1}-\frac{1}{(2 n-1) !} \int_{0}^{\infty} d y \ln y \frac{d^{2 n}}{d y^{2 n}} f(a, y)
$$

From Eq. (B2), we can see that the terms with $x^{4 n}(n \geq 0)$ in $F(a, x)$ do not contribute to $g(a, b)$, so we can express $F(a, x)$ as follows:

$$
\begin{aligned}
F(a, x)= & \frac{1}{4} x^{2} \ln x^{2}+\left(\frac{1}{4}-\frac{1}{2} \ln 2+\frac{1}{2} C_{1}(a)\right) x^{2} \\
& +\sum_{n=1}^{\infty} \frac{(4 n+1) ! !}{(4 n+2) ! !} C_{2 n+1}(a) x^{4 n+2} \\
& +\sum_{n=0}^{\infty}\left(\text { terms with } x^{4 n}\right) .
\end{aligned}
$$

Substituting Eq. (B15) into Eq. (B2), we have

$$
\begin{aligned}
g(a, b)= & \left(\frac{7 \pi^{2}}{360}+\frac{a^{2}}{12}+\frac{a^{4}}{24 \pi^{2}}\right)-\frac{b^{2} \ln b^{2}}{384 \pi^{2}}-\frac{b^{2}}{96 \pi^{2}} \ln \left(\frac{e^{1+C_{1}(a)}}{2 G^{6}}\right) \\
& -\frac{1}{2 \pi^{2}} \sum_{n=1}^{\infty} \frac{(4 n+1) ! !}{(4 n+4) ! !} \mathcal{B}_{2 n+2} C_{2 n+1}(a) b^{2 n+2},
\end{aligned}
$$

where we have used the following integrations:

$$
\begin{gathered}
\int_{0}^{\infty} d t \frac{t \ln t}{e^{2 \pi t}-1}=\frac{1}{24}-\frac{1}{2} \ln G, \\
\int_{0}^{\infty} d t \frac{t^{2 n+1}}{e^{2 \pi t}-1}=(-1)^{n} \frac{\mathcal{B}_{2 n+2}}{4 n+4} \quad(n \geq 0),
\end{gathered}
$$

with Glaisher number $G=1.282427 \ldots$ and Bernoulli numbers $\mathcal{B}_{n}$ defined as

$$
\frac{t}{e^{t}-1}=\sum_{n=0}^{\infty} \frac{\mathcal{B}_{n}}{n !} t^{n}
$$

We list some Bernoulli numbers $\mathcal{B}_{2 n+2}$ as follows:

$\mathcal{B}_{2}=\frac{1}{6}, \quad \mathcal{B}_{4}=-\frac{1}{30}, \quad \mathcal{B}_{6}=\frac{1}{42}, \quad \mathcal{B}_{8}=-\frac{1}{30}$ 


\section{APPENDIX C: ASYMPTOTIC BEHAVIOR \\ OF $C_{2 n+1}(a)$ AS $a \rightarrow \infty$}

To study the asymptotic behavior of $C_{2 n+1}(a)$ as $a \rightarrow \infty$, we rewrite $C_{2 n+1}(a)$ as

$$
\begin{aligned}
C_{2 n+1}(a)= & -\delta_{n, 0}+\frac{1}{(4 n+1) !} \frac{d^{4 n+1}}{d a^{4 n+1}} \\
& \times \int_{0}^{\infty} d y \ln y\left(\frac{1}{e^{y+a}+1}-\frac{1}{e^{y-a}+1}\right) .
\end{aligned}
$$

Making use of the following integration formula:

$$
\begin{aligned}
& \int_{0}^{\infty} d y \ln y\left(\frac{1}{e^{y+a}+1}-\frac{1}{e^{y-a}+1}\right) \\
& =\gamma a+\left\{\frac{d}{d s}\left[\operatorname{Li}_{s}\left(-e^{a}\right)-\operatorname{Li}_{s}\left(-e^{-a}\right)\right]\right\}_{s=1},
\end{aligned}
$$

we have

$$
\begin{aligned}
C_{2 n+1}(a)= & (\gamma-1) \delta_{n, 0}+\frac{1}{(4 n+1) !} \frac{d^{4 n+1}}{d a^{4 n+1}} \\
& \times\left\{\frac{d}{d s}\left[\operatorname{Li}_{s}\left(-e^{a}\right)-\operatorname{Li}_{s}\left(-e^{-a}\right)\right]\right\}_{s=1},
\end{aligned}
$$

where $\operatorname{Li}_{s}(z)$ is the polylogarithm function defined as

$$
\mathrm{Li}_{s}(z)=\sum_{k=1}^{\infty} \frac{z^{k}}{k^{s}}
$$

This definition of $\operatorname{Li}_{s}(z)$ is valid for arbitrary complex number $s$ and for $|z|<1$, and it can be extended to $|z| \geq 1$ by the process of analytic continuation. Making use of the following asymptotic formulas for $\operatorname{Li}_{s}(z)$ :

$$
\operatorname{limLi}_{z \rightarrow 0}(z)=z
$$

$\lim _{a \rightarrow \infty} \operatorname{Li}_{s}\left(-e^{a}\right)=-\frac{a^{s}}{\Gamma(s+1)} \quad(s \neq-1,-2,-3, \ldots)$,

we have

$$
\lim _{a \rightarrow \infty}\left[\frac{d}{d s}\left(\operatorname{Li}_{s}\left(-e^{a}\right)-\operatorname{Li}_{s}\left(-e^{-a}\right)\right)\right]_{s=1}=(1-\gamma) a-a \ln a,
$$

where we have used $\Gamma^{\prime}(2)=1-\gamma$. So we obtain

$$
\begin{aligned}
\lim _{a \rightarrow \infty} C_{2 n+1}(a)= & (\gamma-1) \delta_{n, 0} \\
& +\frac{1}{(4 n+1) !} \frac{d^{4 n+1}}{d a^{4 n+1}}[(1-\gamma) a-a \ln a] \\
= & -\frac{1}{(4 n+1) !} \frac{d^{4 n+1}}{d a^{4 n+1}}(a \ln a) \\
= & \begin{cases}-1-\ln a, & n=0, \\
\frac{1}{4 n(4 n+1)} a^{-4 n}, & n>0,\end{cases}
\end{aligned}
$$

which shows that $C_{1}(a) \rightarrow-\infty$ and $C_{2 n+1}(a) \rightarrow 0(n>0)$ as $a \rightarrow \infty$. Furthermore, we have

$$
\lim _{a \rightarrow \infty} C_{2 n+1}^{\prime}(a)=-\frac{1}{4 n+1} a^{-4 n-1} \rightarrow 0(n \geq 0) .
$$

\section{APPENDIX D: EXPANSION OF $C_{2 n+1}(a)$ AT $a=0$}

To expand $C_{2 n+1}(a)$ at $a=0$, we use the following expression of $C_{2 n+1}(a)$ :

$$
\begin{aligned}
C_{2 n+1}(a)= & -\delta_{n, 0}+\frac{1}{(4 n+1) !} \int_{0}^{\infty} d y \ln y \frac{d^{4 n+1}}{d y^{4 n+1}} \\
& \times\left(\frac{1}{e^{y+a}+1}+\frac{1}{e^{y-a}+1}\right) .
\end{aligned}
$$

When $n>0$, we have

$$
\begin{aligned}
C_{2 n+1}(a)= & \frac{2}{(4 n+1) !} \int_{0}^{\infty} d y \ln y \frac{d^{4 n+1}}{d y^{4 n+1}} \\
& \times \sum_{k=0}^{\infty} \frac{a^{2 k}}{(2 k) !} \frac{d^{2 k}}{d y^{2 k}}\left(\frac{1}{e^{y}+1}\right) \\
= & -\frac{2}{(4 n+1) !} \sum_{k=0}^{\infty} \frac{a^{2 k}}{(2 k) !} \\
& \times \int_{0}^{\infty} d y \frac{1}{y} \frac{d^{4 n+2 k}}{d y^{4 n+2 k}}\left(\frac{1}{e^{y}+1}\right) .
\end{aligned}
$$

In order to calculate the integration in the second line of Eq. (D2), we make use of the following identities:

$$
\begin{gathered}
\Gamma(s) \operatorname{Li}_{s}\left(-e^{x}\right)=-\int_{0}^{\infty} d y \frac{1}{y^{1-s}} \frac{1}{e^{y-x}+1}(\operatorname{Re} s>0), \quad(\mathrm{D} 3) \\
\frac{d}{d x} \operatorname{Li}_{s}\left(-e^{x}\right)=\operatorname{Li}_{s-1}\left(-e^{x}\right), \\
\lim _{s \rightarrow 0} \Gamma(s) \operatorname{Li}_{s-2 n}(-1)=\left(2^{2 n+1}-1\right) \zeta^{\prime}(-2 n) \quad(n=1,2,3, \ldots),
\end{gathered}
$$

and then we can obtain 


$$
\begin{aligned}
& \int_{0}^{\infty} d y \frac{1}{y} \frac{d^{4 n+2 k}}{d y^{4 n+2 k}}\left(\frac{1}{e^{y}+1}\right) \\
& \quad=\lim _{s \rightarrow 0} \int_{0}^{\infty} d y \frac{1}{y^{1-s}}\left(\frac{d^{4 n+2 k}}{d x^{4 n+2 k}} \frac{1}{e^{y-x}+1}\right)_{x=0} \\
& \quad=-\lim _{s \rightarrow 0} \Gamma(s)\left[\frac{d^{4 n+2 k}}{d x^{4 n+2 k}} \mathrm{Li}_{s}\left(-e^{x}\right)\right]_{x=0} \\
& =-\lim _{s \rightarrow 0} \Gamma(s) \mathrm{Li}_{s-4 n-2 k}(-1) \\
& =\left(1-2^{4 n+2 k+1}\right) \zeta^{\prime}(-4 n-2 k) .
\end{aligned}
$$

So Eq. (D2) becomes

$$
C_{2 n+1}(a)=\frac{2}{(4 n+1) !} \sum_{k=0}^{\infty}\left(2^{4 n+2 k+1}-1\right) \zeta^{\prime}(-4 n-2 k) \frac{a^{2 k}}{(2 k) !} .
$$

When $n=0$ in Eq. (D1), a similar calculation gives

$$
C_{1}(a)=\ln 4+\gamma-1+2 \sum_{k=0}^{\infty}\left(2^{2 k+1}-1\right) \zeta^{\prime}(-2 k) \frac{a^{2 k}}{(2 k) !} .
$$

The general expression of $C_{2 n+1}(a)$ for $n \geq 0$ is

$$
\begin{aligned}
C_{2 n+1}(a)= & (\ln 4+\gamma-1) \delta_{n, 0}+\frac{2}{(4 n+1) !} \\
& \times \sum_{k=0}^{\infty}\left(2^{4 n+2 k+1}-1\right) \zeta^{\prime}(-4 n-2 k) \frac{a^{2 k}}{(2 k) !} .
\end{aligned}
$$

Especially, when $a=0$, we have

$$
C_{2 n+1}(0)=(\ln 4+\gamma-1) \delta_{n, 0}+\frac{2 \zeta^{\prime}(-4 n)}{(4 n+1) !}\left(2^{4 n+1}-1\right) .
$$

\section{APPENDIX E: ASYMPTOTIC BEHAVIOR OF $g(a, b)$ AS $b \rightarrow \infty$}

To study the asymptotic behavior of $g(a, b)$ as $b \rightarrow \infty$, we may rewrite $g(a, b)$ in Eq. (23) as

$$
\begin{aligned}
g(a, b)= & \left(\frac{7 \pi^{2}}{360}+\frac{a^{2}}{12}+\frac{a^{4}}{24 \pi^{2}}\right)+\frac{1}{4 \pi^{2}} \times i \int_{0}^{\infty} d s \\
& \times \int_{0}^{\infty} d t \frac{f\left(a, \sqrt{i t+s^{2}}\right)-f\left(a, \sqrt{-i t+s^{2}}\right)}{e^{2 \pi t / b}-1},
\end{aligned}
$$

where $f(a, x)=\ln \left(1+e^{a-x}\right)+\ln \left(1+e^{-a-x}\right)$ as defined in Eq. (20). As $z \rightarrow 0$, we can expand $1 /\left(e^{z}-1\right)$ as follows:

$$
\frac{1}{e^{z}-1}=\frac{1}{z}-\frac{1}{2}+\frac{z}{12}-\frac{z^{3}}{720}+\frac{z^{5}}{30240}+\cdots
$$

So as $b \rightarrow \infty$ (i.e., $1 / b \rightarrow 0), g(a, b)$ becomes

$$
\begin{aligned}
g(a, b)= & \left(\frac{7 \pi^{2}}{360}+\frac{a^{2}}{12}+\frac{a^{4}}{24 \pi^{2}}\right)+\frac{b}{8 \pi^{3}} \times i \int_{0}^{\infty} d t \\
& \times \frac{1}{t} \int_{0}^{\infty} d s\left[f\left(a, \sqrt{i t+s^{2}}\right)-f\left(a, \sqrt{-i t+s^{2}}\right)\right] \\
& -\frac{1}{8 \pi^{2}} \times i \int_{0}^{\infty} d t \int_{0}^{\infty} d s\left[f\left(a, \sqrt{i t+s^{2}}\right)\right. \\
& \left.-f\left(a, \sqrt{-i t+s^{2}}\right)\right]+\mathcal{O}\left(\frac{1}{b}\right) .
\end{aligned}
$$

Fortunately, the two integrations in Eq. (E3) can be analytically integrated out as follows:

$$
\begin{aligned}
& i \int_{0}^{\infty} d t \frac{1}{t} \int_{0}^{\infty} d s\left[f\left(a, \sqrt{i t+s^{2}}\right)-f\left(a, \sqrt{-i t+s^{2}}\right)\right] \\
& =\frac{\pi^{3}}{6}+\frac{\pi}{2} a^{2} \\
& i \int_{0}^{\infty} d t \int_{0}^{\infty} d s\left[f\left(a, \sqrt{i t+s^{2}}\right)-f\left(a, \sqrt{-i t+s^{2}}\right)\right] \\
& =\frac{7 \pi^{4}}{45}+\frac{2 \pi^{2}}{3} a^{2}+\frac{1}{3} a^{4} .
\end{aligned}
$$

So we have

$$
\lim _{b \rightarrow \infty} g(a, b)=\frac{b}{48 \pi^{2}}\left(\pi^{2}+3 a^{2}\right) .
$$

\section{APPENDIX F: NORMAL ORDERING AND UN-NORMAL ORDERING}

The explicit form of energy density $\varepsilon$ expressed by a summation of all quantum states in Eq. (39) can also be derived from the ensemble average of normal ordering (NO) of the energy density operator as described in Sec. VI.

If we adopt un-normal ordering (UNO), then Eqs. (57) and (59) become, respectively,

$$
\begin{array}{r}
\left\langle\theta\left(-k_{z}\right) b_{0}\left(k_{y}, k_{z}\right) b_{0}^{\dagger}\left(k_{y}, k_{z}\right)\right\rangle=\theta\left(-k_{z}\right)\left[1-\frac{1}{e^{\beta\left(-k_{z}+\mu_{R}\right)}+1}\right], \\
(\mathrm{F} 1) \\
\left\langle b_{n}\left(k_{y}, k_{z}\right) b_{n}^{\dagger}\left(k_{y}, k_{z}\right)\right\rangle=1-\frac{1}{e^{\beta\left[E_{n}\left(k_{z}\right)+\mu_{R}\right]}+1} \cdot \quad(\mathrm{F} 2)
\end{array}
$$

This UNO description is also used in recent articles [54,55]. Now the ensemble average of the energy density operator becomes 


$$
\begin{aligned}
\varepsilon_{\mathrm{un}}= & \frac{1}{V} \sum_{k_{y}, k_{z}}\left[k_{z} \theta\left(k_{z}\right) \frac{1}{e^{\beta\left(k_{z}-\mu_{R}\right)}+1}\right. \\
& \left.+\left(-k_{z}\right) \theta\left(-k_{z}\right)\left(\frac{1}{e^{\beta\left(-k_{z}+\mu_{R}\right)}+1}-1\right)\right] \\
& +\frac{1}{V} \sum_{n=1}^{\infty} \sum_{k_{y}, k_{z}} E_{n}\left(k_{z}\right)\left[\frac{1}{e^{\beta\left[E_{n}\left(k_{z}\right)-\mu_{R}\right]}+1}\right. \\
& \left.+\frac{1}{e^{\beta\left[E_{n}\left(k_{z}\right)+\mu_{R}\right]}+1}-1\right]
\end{aligned}
$$

where the subscript "un" of $\varepsilon_{\text {un }}$ means un-normal ordering. We can see that the UNO description leads to the infinite vacuum energy in $\varepsilon_{\text {un }}$. Comparing Eqs. (39) and (F3), it is equivalent to do the following regularization:

$$
\varepsilon_{\text {un }}=\varepsilon-\lim _{\mu_{R} \rightarrow \infty} \varepsilon
$$

Making use of two dimensionless variables $a=\beta \mu_{R}$ and $b=2 e B \beta^{2}$ instead of $\mu_{R}, \beta$, and $e B$, Eq. (F4) can be rewritten as

$$
\varepsilon_{\text {un }}(a, b)=\varepsilon(a, b)-\lim _{a \rightarrow \infty} \varepsilon(a, b) .
$$

From the expression of $\varepsilon(a, b)$ in Eq. (29), Eq. (F5) becomes

$$
\begin{aligned}
\varepsilon_{\mathrm{un}}(a, b)= & \varepsilon_{0}+\frac{1}{2 \pi^{2} \beta^{4}}\left[\left(\frac{\pi^{2} a^{2}}{2}+\frac{a^{4}}{4}\right)\right. \\
& \left.+\sum_{n=0}^{\infty} \frac{(4 n+1) ! !}{(4 n+4) ! !}(4 n+1) \mathcal{B}_{2 n+2} C_{2 n+1}^{\mathrm{un}}(a) b^{2 n+2}\right],
\end{aligned}
$$

where $C_{2 n+1}^{\mathrm{un}}(a)$ is defined as

$$
C_{2 n+1}^{\mathrm{un}}(a)=C_{2 n+1}(a)-\lim _{a \rightarrow \infty} C_{2 n+1}(a)
$$

and $\varepsilon_{0}$ is just the familiar infinite vacuum energy, as in the free field theory $[42,56]$. This infinite vacuum energy cannot be detected experimentally, since only the energy difference from the ground state can be observable. It is worth pointing out that the term $b^{2} \ln b^{2}$ in $\varepsilon$ disappears in $\varepsilon_{\text {un }}$ and the $b^{2}$ order of $\varepsilon_{\text {un }}$ is consistent with Ref. [35].

In Eq. (C8), we obtained the asymptotic behavior of $C_{2 n+1}(a)$ as $a \rightarrow \infty$ :

$$
\lim _{a \rightarrow \infty} C_{2 n+1}(a)= \begin{cases}-1-\ln a, & n=0, \\ \frac{1}{4 n(4 n+1)} a^{-4 n}, & n>0,\end{cases}
$$

which shows that $C_{1}(a) \rightarrow-\infty$ and $C_{2 n+1}(a) \rightarrow 0(n>0)$ as $a \rightarrow \infty$. So we have $C_{2 n+1}^{\text {un }}(a)=C_{2 n+1}(a)$ for $n>0$. For $n=0$, another expression of $C_{1}(a)$ is useful:

$$
C_{1}(a)=\gamma-1-\int_{0}^{\infty} d y \frac{1}{y}\left(\frac{1}{e^{y-a}+1}+\frac{1}{e^{y+a}+1}-\frac{1}{e^{y}}\right),
$$

which leads to

$$
C_{1}^{\mathrm{un}}(a)=\int_{0}^{\infty} d y \frac{1}{y}\left(1-\frac{1}{e^{y-a}+1}-\frac{1}{e^{y+a}+1}\right) .
$$

Note that $C_{1}^{\mathrm{un}}(a)$ is a positive infinity, which is expected, since $C_{1}(a) \rightarrow-\infty$ as $a \rightarrow \infty$. This expression of $C_{1}^{\mathrm{un}}(a)$ can also be obtained in Ref. [35], where the authors adopt dimensional regularization to deal with the divergence and obtain a regular term $\ln \left(\Lambda^{2} / T^{2}\right)$ instead of the singular term $\ln \left(e B / T^{2}\right)$ in the energy density $\varepsilon_{\text {un }}$, with the renormalization scale $\Lambda$.

Since only the derivative of $C_{2 n+1}(a)$ appears in the expression of the particle number density $n$ in Eq. (28), and $C_{2 n+1}^{\prime}(a) \rightarrow 0$ as $a \rightarrow \infty$ as calculated in Eq. (C9), i.e., $C_{2 n+1}^{\mathrm{un} \prime}(a)=C_{2 n+1}^{\prime}(a)$, the UNO and NO descriptions can obtain the same expression of the particle number density $n$, which is also consistent with Ref. [35].
[1] J. Schwinger, Phys. Rev. 82, 664 (1951).

[2] S.P. Kim and D. N. Page, Phys. Rev. D 65, 105002 (2002).

[3] E. Brezin and C. Itzykson, Phys. Rev. D 2, 1191 (1970).

[4] Z.-q. Zhang, D.-f. Hou, and G. Chen, Eur. Phys. J. A 53, 51 (2017).

[5] X.-L. Sheng, R.-H. Fang, Q. Wang, and D. H. Rischke, Phys. Rev. D 99, 056004 (2019).
[6] Z.-R. Zhu, D.-f. Hou, and X. Chen, Eur. Phys. J. C 80, 550 (2020).

[7] R. Felipe, A. Martinez, H. Rojas, and M. Orsaria, Phys. Rev. C 77, 015807 (2008).

[8] A. Reisenegger, in International Workshop on Strong Magnetic Fields and Neutron Star (2003), pp. 33-49, arXiv:astro-ph/0307133.

[9] K. Itokazu, K. Yanase, and N. Yoshinaga, J. Phys. Soc. Jpn. Conf. Proc. 23, 013003 (2018). 
[10] A. Reisenegger, arXiv:1305.2542.

[11] V. Skokov, A. Illarionov, and V. Toneev, Int. J. Mod. Phys. A 24, 5925 (2009).

[12] A. Bzdak and V. Skokov, Phys. Lett. B 710, 171 (2012).

[13] V. Voronyuk, V. Toneev, W. Cassing, E. Bratkovskaya, V. Konchakovski, and S. Voloshin, Phys. Rev. C 83, 054911 (2011).

[14] W.-T. Deng and X.-G. Huang, Phys. Rev. C 85, 044907 (2012).

[15] D. E. Kharzeev, L. D. McLerran, and H. J. Warringa, Nucl. Phys. A803, 227 (2008).

[16] K. Fukushima, D. E. Kharzeev, and H. J. Warringa, Phys. Rev. D 78, 074033 (2008).

[17] J.-H. Gao, Z.-T. Liang, S. Pu, Q. Wang, and X.-N. Wang, Phys. Rev. Lett. 109, 232301 (2012).

[18] B. Feng, D.-F. Hou, and H.-C. Ren, Phys. Rev. D 99, 036010 (2019).

[19] G.-R. Liang, J. Liao, S. Lin, L. Yan, and M. Li, arXiv: 2004.04440.

[20] V. Gusynin, V. Miransky, and I. Shovkovy, Phys. Lett. B 349, 477 (1995).

[21] K. Fukushima and J. M. Pawlowski, Phys. Rev. D 86, 076013 (2012).

[22] G. Bali, F. Bruckmann, G. Endrodi, F. Gruber, and A. Schaefer, J. High Energy Phys. 04 (2013) 130.

[23] V. A. Miransky and I. A. Shovkovy, Phys. Rep. 576, 1 (2015).

[24] S. Mao, Y. Wu, and P. Zhuang, J. Phys. Soc. Jpn. Conf. Proc. 20, 011009 (2018).

[25] A. Ballon-Bayona, J. P. Shock, and D. Zoakos, arXiv: 2005.00500.

[26] G. Cao and X.-G. Huang, Phys. Lett. B 757, 1 (2016).

[27] R.-h. Fang, J.-y. Pang, Q. Wang, and X.-n. Wang, Phys. Rev. D 95, 014032 (2017).

[28] H.-L. Chen, X.-G. Huang, and K. Mamed, arXiv: 1910.02700 .

[29] L. Wang, G. Cao, X.-G. Huang, and P. Zhuang, Phys. Lett. B 780, 273 (2018).

[30] D. Cangemi and G. V. Dunne, Ann. Phys. (N.Y.) 249, 582 (1996).

[31] X.-G. Huang, M. Huang, D. H. Rischke, and A. Sedrakian, Phys. Rev. D 81, 045015 (2010).

[32] J.-W. Chen, S. Pu, Q. Wang, and X.-N. Wang, Phys. Rev. Lett. 110, 262301 (2013).

[33] Y. Hidaka, S. Pu, and D.-L. Yang, Phys. Rev. D 95, 091901 (2017).
[34] J.-H. Gao, Z.-T. Liang, Q. Wang, and X.-N. Wang, Phys. Rev. D 98, 036019 (2018).

[35] S.-Z. Yang, J.-H. Gao, Z.-T. Liang, and Q. Wang, arXiv: 2003.04517.

[36] E. Gorbar, V. Miransky, I. Shovkovy, and P. Sukhachov, Phys. Rev. B 95, 205141 (2017).

[37] S. Lin and L. Yang, Phys. Rev. D 101, 034006 (2020).

[38] R.-D. Dong, R.-H. Fang, D.-F. Hou, and D. She, Chin. Phys. C 44, 074106 (2020).

[39] W. Dittrich, Phys. Rev. D 19, 2385 (1979).

[40] H. Gies, Phys. Rev. D 60, 105002 (1999).

[41] S. Ozaki, T. Arai, K. Hattori, and K. Itakura, Phys. Rev. D 92, 016002 (2015).

[42] M. Peskin and D. Schroeder, An Introduction to Quantum Field Theory (Westview Press, New York, 1995).

[43] G.-J. Ni and S.-Q. Chen, Advanced Quantum Mechanics, 2nd ed. (Fudan University Press, Shanghai, 2003).

[44] P. L. Butzer, P. J. S. G. Ferreira, G. Schmeisser, and R. L. Stens, Results Math. 59, 359 (2011).

[45] G. S. Bali, G. Endrődi, and S. Piemonte, J. High Energy Phys. 07 (2020) 183.

[46] C. Bonati, M. D'Elia, M. Mariti, F. Negro, and F. Sanfilippo, Proc. Sci. LATTICE2013 (2014) 184 [arXiv:1312.5070].

[47] I. Arsene et al. (BRAHMS Collaboration), Nucl. Phys. A757, 1 (2005).

[48] J. Adams et al. (STAR Collaboration), Nucl. Phys. A757, 102 (2005).

[49] K. Adcox et al. (PHENIX Collaboration), Nucl. Phys. A757, 184 (2005).

[50] M. Ishihara and F. Takagi, Phys. Rev. C 59, 2221 (1999).

[51] M. Ishihara and F. Takagi, Phys. Rev. C 61, 024903 (1999).

[52] D. Vasak, M. Gyulassy, and H. T. Elze, Ann. Phys. (N.Y.) 173, 462 (1987).

[53] G. V. Dunne, in Heisenberg-Euler Effective Lagrangians: Basics and Extensions (2004), pp. 445-522, arXiv:hep-th/ 0406216.

[54] X.-l. Sheng, D. H. Rischke, D. Vasak, and Q. Wang, Eur. Phys. J. A 54, 21 (2018).

[55] J.-H. Gao, Z.-T. Liang, and Q. Wang, Phys. Rev. D 101, 096015 (2020).

[56] J. Kapusta and C. Gale, Finite-Temperature Field Theory: Principles and Applications, 2nd ed. (Cambridge University Press, Cambridge, England, 2006). 